\title{
Desenvolvimento Psicomotor de Crianças com Baixo Peso
}

\author{
Marilia Rocha de Sousa, ${ }^{1}$ Zélia Ferreira Caçador Anastácio, ${ }^{2}$ \\ Andrea Stopiglia Guedes Braide ${ }^{3}$
}

\begin{abstract}
RESUMO
Objetivo: Avaliar o desenvolvimento psicomotor de crianças com baixo peso. Metodologia: Estudo de campo, descritivo, observacional, transversal e com abordagem quantitativa, realizado no período de abril a julho de 2017 , no Instituto da Primeira Infância. Foi aprovado pelo parecer 2.007.070/2017, seguindo a Resolução 466/12. Foram avaliadas 34 crianças com a faixa etária de 4 a 6 anos, utilizando a Bateria Psicomotora de Vitor da Fonseca - adaptada. Os dados foram analisados pelo software Statistical Packcage for the Social Sciences versão 20.0. Resultados: Predomínio do gênero feminino, da faixa etária de 6 anos e da renda mensal de até 1 salário mínimo. Do total de participantes, observou-se que $20(59,8 \%)$ apresentaram o perfil dispráxico, $12(35,2 \%)$ o perfil normal e dois $(5,9 \%)$ o perfil bom. Correlacionando os fatores psicomotores com o perfil observou-se que, com exceção da tonicidade, todos os outros seis fatores (equilíbrio, lateralidade, noção de corpo, estruturação espaçotemporal, praxia global e fina) obtiveram valores com a prevalência de pontuação 1 e 2 , definindo um déficit psicomotor decorrente do baixo peso. Foi constatado também que $16(57,1 \%)$ crianças, mesmo com a recuperação do estado nutricional, ainda apresentavam o perfil dispráxico. Conclusão: É possível, por meio de ações conjuntas de acompanhamento nutricional e intervenção da psicomotricidade, restabelecer o déficit psicomotor e favorecer uma intervenção direcionada para o desenvolvimento psicomotor da criança.
\end{abstract}

Palavras-chave: Desenvolvimento. Criança. Desempenho psicomotor.

\section{PSYCHOMOTOR DEVELOPMENT OF LOW WEIGHT CHILDREN}

\section{ABSTRACT}

Objective: To evaluate the psychomotor development of children with low weight. Methods: Field study, descriptive, observational, transversal and with quantitative approach, carried out from april to july 2017, in early childhood institute. It was approved by the 2.007.070/2017, following Resolution 466/12. Were evaluated 34 children aged 4 to 6 years, using the Psychomotor Battery in Vitor da Fonseca - adapted. The data were analyzed by software Statistical Packcage for the Social Sciences version 20.0. Results: Prevalence of the female gender, the age group of six years and the monthly income of up to one minimum wage. From the total of participants, it was observed that $20(59,8 \%)$ presented the dyspraxic profile, $12(35,2 \%)$ the normal profile and two $(5,9 \%)$ the good profile. Correlating the psychomotor factors with the profile, it was observed that, with the exception of tonicity, all other six factors (balance, laterality, body notion, spatio-temporal structuring, global and fine praxis) obtained values with the prevalence of scores 1 and 2, defining a psychomotor deficit due to low weight. It was also found that $16(57,1 \%)$ children, even with recovery of nutritional status, still presented the dyspraxic profile. Conclusions: It is possible through joint actions of nutritional monitoring and intervention of psychomotricity, reestablish the low weight and favor an intervention directed to the psychomotor development of the child.

Keywords: Development. Child. Psychomotor performance.

\footnotetext{
${ }^{1}$ Autora correspondente. Centro Universitário Christus. Rua Padre Guerra - 2735, Parquelândia, CEP 60440-605, Fortaleza, CE, Brasil. http://lattes.cnpq.br/ 6216923045420149. https://orcid.org/0000-0003-4599-3280.mariliarocha.sousa@hotmail.com

2 Universidade do Minho - Campus de Gualtar. Braga, Portugal.

${ }^{3}$ Escola de Saúde Pública do Ceará. Fortaleza/CE, Brasil.
} 


\section{INTRODUÇÃO}

Desde a concepção no útero materno até ao momento em que morre, o ser humano passa por um processo de grandes mudanças. Este processo, que resulta da interação entre as características biológicas de cada indivíduo, é denominado de desenvolvimento humano (DIAS; CORREIA; MARCELINO, 2013).

De acordo com o Programa das Nações Unidas para o Desenvolvimento (PNUD, 2017), o desenvolvimento humano é definido como um processo de ampliação das escolhas das pessoas para que elas tenham capacidades e oportunidades, na qual é necessário considerar características sociais, culturais e políticas que influenciam na qualidade de vida humana. Araújo (2013) considera que os primeiros anos de vida de uma criança são de fundamental importância para o seu desenvolvimento, pois é nesse período que ocorre a maior plasticidade neural, caracterizada pela capacidade do Sistema Nervoso Central de reorganizar e autorreparar/ autoadaptar as redes neuronais.

Baseado em Portugal (2009), o período da infância e as primeiras experiências de vida do ser humano ainda nesta fase determinam aquilo que ele será quando adulto, pois é nesse período que o sujeito aprende sobre si, sobre o outro e sobre o mundo.

Portugal (2009) reconhece que quando existe a carência de estimulação, seja ela motora, cognitiva ou nutricional, normalmente pode ocorrer um déficit do desenvolvimento psicomotor da criança, pois é nesse período que ela se encontra em uma situação de vulnerabilidade e que se faz necessária uma intervenção precoce para a prevenção de distúrbios posteriores. O estudo de Ferreira, Martinez e Ciasca (2010) apresenta o desenvolvimento psicomotor como a aquisição funcional de todo o corpo da criança, tendo como foco principal o controle do próprio corpo e a capacidade de extrair todas as possibilidades de ação e expressão possíveis. Este desenvolvimento, na visão destes autores, progride lentamente e está relacionado com as experiências e oportunidades que a criança possui em explorar o ambiente no qual está inserida. Quando não ocorre um desenvolvimento adequado, identifica-se, normalmente, um déficit das habilidades motoras que pode ser decorrente da carência dessas vivências corporais.

Fonseca (2008) identifica que várias causas podem provocar um atraso no desenvolvimento da criança, e esse atraso, caso não seja diagnosticado precocemente e não seja tratado, pode gerar comprometimentos a médio e longo prazos. Entre as principais causas de atraso do desenvolvimento encon- tram-se: baixo peso ao nascer, desnutrição, distúrbios cardiovasculares, respiratórios e neurológicos, infecções neonatais, baixas condições socioeconômicas, nível educacional precário dos pais, prematuridade e as malformações congênitas (WILLRICH et al., 2009).

De acordo com os dados apresentados nos estudos de Caçola e Bobbio (2010) e de Maia e Souza (2010), o baixo peso ao nascer (BPN) é definido como o peso inferior a $2.500 \mathrm{~g}$. Os autores consideram que essa condição é um dos principais preditores de morbimortalidade neonatal e perinatal, tanto em países desenvolvidos quanto em desenvolvimento. Ainda reforçam estes autores que quando o BPN está associado à nutrição precária, ao alto índice de infecção e a outras condições de pobreza, resulta em alterações cognitivas e comportamentais significativas para a criança. Isto pode revelar a lentidão e déficits no processo do desenvolvimento de algumas crianças nascidas com baixo peso e que, mesmo com rigoroso acompanhamento desde os primeiros dias de nascidas, ainda assim apresentam baixa taxa de crescimento ao longo dos primeiros anos de vida.

Está bem estabelecido que crianças com BPN, além da menor oportunidade de sobrevivência, apresentam menores possibilidades de um desenvolvimento neuropsicomotor adequado. Apresentam também uma evolução mental e motora estatisticamente mais baixa, principalmente no primeiro ano de vida, revelando a importância do peso ao nascer no desenvolvimento. Somado a isso, o BPN é um dos principais agentes responsáveis pelo risco nutricional ao final do primeiro ano de vida, sendo imprescindível adotar estratégias para sua redução e prevenção, para que não haja prejuízo maior durante a primeira infância (MAYER; CANCELIER; FRANCIOTTI, 2011).

Há evidências de que essas crianças apresentam déficits em diversas áreas do desenvolvimento, incluindo alterações de coordenação motora, atenção, desempenho acadêmico e comportamental, além de comprometimento na estatura e deficiência nutricional (OLIVEIRA; MAGALHÃES; SALMELA, 2011). Diante do exposto, o estudo teve como objetivo avaliar o desenvolvimento psicomotor das crianças com baixo peso, identificando as possíveis alterações apresentadas por elas e possibilitando uma intervenção.

\section{METODOLOGIA}

Foi realizado um estudo de campo, descritivo, observacional, documental transversal e com abordagem quantitativa, no período de abril a julho de 2017, no Instituto da Primeira Infância - Iprede - lo- 
calizado no Estado do Ceará - Brasil. Esta instituição desenvolve ações intervencionistas relacionadas ao acompanhamento nutricional e ao desenvolvimento neuropsicomotor nos primeiros anos de vida da criança. Interage ainda com os familiares que acompanham essas crianças e que apresentam situações de vulnerabilidade social (INSTITUTO..., 2017).

As famílias assistidas na instituição apresentam a característica do desfavorecimento financeiro e, desse modo, encontram-se em uma situação de vulnerabilidade social. São assim classificadas por se encontrarem diretamente ligadas à pobreza estrutural, agravada pela situação socioeconômica. Devido a isso, apresentam grandes dificuldades de proporcionar uma condição alimentar de qualidade aos seus fiIhos, desencadeando o baixo peso, com necessidade de suporte, orientação e intervenção especializada no desenvolvimento neuropsicomotor ainda na primeira infância (GABBARD, 2016; JARDIM, 2015).

Este estudo iniciou-se após aprovação pelo Comitê de Ética em Pesquisa do Centro Universitário Christus sob o parecer 2.007.070/2017, seguindo os princípios éticos de pesquisa envolvendo seres humanos da Resolução 466/12 do Conselho Nacional de Saúde. Ao ser admitida no Iprede a criança apresenta uma condição nutricional desfavorável desde o nascimento e mantém a condição de baixo peso durante o período da primeira infância, necessitando de uma assistência direcionada para reverter o quadro de peso insuficiente.

O perfil nutricional dessas crianças é traçado por protocolo de triagem a partir do acolhimento na instituição. Leva-se em consideração os parâmetros antropométricos utilizados no programa da Política Nacional de Alimentação e Nutrição (PNAN), que acompanha os males relacionados à escassez alimentar no foco da desnutrição infantil (BRASIL, 2013).

As crianças participantes deste estudo foram classificadas no nascimento com baixo peso $(100 \%$ da população para inclusão neste estudo) e então, encaminhadas desde a alta hospitalar para regular acompanhamento de puericultura na Unidade Básica de Saúde local. Quando realizado o encaminhamento para uma unidade de saúde especializada - Iprede - essas crianças são acolhidas e avaliadas por uma equipe multidisciplinar, que tem como objetivo acompanhar, por meio de ações de promoção do crescimento e desenvolvimento, o quadro inicial em que se encontra, com ênfase no contexto social, econômico e cultural.
Durante o período de acompanhamento da criança é realizada periodicamente a verificação de peso e estatura antes de qualquer atendimento, para a atualização da puericultura, e assim verificar a sua evolução.

Para iniciar a coleta de dados foram realizadas as análises de prontuários das crianças que nasceram com baixo peso e que faziam acompanhamento no Iprede e que estavam sendo atendidas no Setor de Psicomotricidade da instituição. Foram incluídas na pesquisa crianças de ambos os gêneros, na faixa etária de 4 a 6 anos, sob acompanhamento na instituição pelo diagnóstico inicial de baixo peso e que são atendidas no Setor de Psicomotricidade. 0 estudo teve uma população de 50 crianças, das quais 34 estavam dentro da faixa etária estipulada e foram incluídas. A amostra da pesquisa foi selecionada por cálculo amostral e pelo método de aleatorização simples de acordo com o quantitativo de crianças em atendimento no setor, no período anteriormente indicado.

Pelo fato de a pesquisa ter sido realizada com crianças de 4 a 6 anos, os responsáveis foram abordados e esclarecidos sobre os objetivos e a justificativa do estudo, assinando o Termo de Consentimento Livre e Esclarecido, autorizando a participação da criança. Foi utilizado inicialmente para coleta de dados um questionário no qual constavam os dados de identificação da criança (número do prontuário, idade, gênero, peso e estatura ao nascer e peso e estatura atual) e dados sociodemográficos do responsável (grau de parentesco, escolaridade, profissão, renda mensal e residência).

Para identificar o perfil psicomotor das crianças foi utilizado como instrumento de coleta de dados a Bateria Psicomotora (BPM) de Vitor da Fonseca - adaptada, na qual constam atividades predeterminadas que devem ser realizadas pelas crianças. A Bateria Psicomotora utilizada como instrumento foi aplicada durante os atendimentos das crianças participantes no Setor de Psicomotricidade, seguindo a demanda normal da instituição. Este instrumento avalia os sete fatores psicomotores, que são: tonicidade, equilibração, lateralização, noção do corpo, estruturação espaçotemporal, praxia global e praxia fina. Após a realização das atividades predeterminadas, é dada uma pontuação pelo examinador de acordo com a execução da tarefa e da escala de pontuação, que varia de 1 a 4 (a pontuação 1 corresponde à realização imperfeita, incompleta e descoordenada; 2 à realização com dificuldades de controle; 3 à realização controlada e adequada e 4 à realização perfeita, econômica, harmoniosa e bem controlada). Ao final da aplicação da BPM é obtida uma 
soma que identifica o perfil psicomotor correspondente. De acordo com a classificação do perfil, os resultados são: superior e bom (não apresenta dificuldades específicas e não deve apresentar em nenhum fator uma pontuação inferior a 3), normal (sem dificuldades específicas, podendo, no entanto, apresentar fatores já mais variados e diferenciados), dispráxico (realizam a tarefa com dificuldades de controle e combinações de sinais desviantes, sendo fácil identificar problemas de equilíbrio, lateralidade e movimentos globais ou finos) e deficitário (apresentam sinais disfuncionais evidentes, não realizam ou realizam de forma imperfeita e incompleta a maioria das tarefas).

Os dados foram exportados para o Microsoft Excel e posteriormente para o software Statistical Packcage for the Social Sciences (SPSS), versão 20.0, no qual as análises foram feitas utilizando estatística descritiva com média e expressando a frequência absoluta e percentual de cada dado, comparado por meio do teste exato de Fisher ou qui-quadrado de Pearson, quando indicado, com uma confiança de $95 \%$. Foi considerada significância estatística um valor de $p<0,05$.

\section{RESULTADOS}

Quanto ao desenvolvimento psicomotor de crianças com baixo peso trazemos resultados pontuais do estudo que puderam responder a alguns questionamentos, identificando as alterações presentes e a possibilidade de uma intervenção adequada, otimizando a recuperação do peso e evitando sequelas funcionais. Participaram do estudo 34 crianças, das quais 18 (52,9\%) eram do gênero feminino, 17 (50\%) estavam na faixa etária de 6 anos e 27 (79,4\%) estavam acompanhadas das mães. Ao ser realizada a busca da caracterização sociodemográfica das famílias acompanhadas no Iprede, obtivemos que 12 acompanhantes (35,3\%) tinham o Ensino Médio completo, 25 (73,5\%) trabalhavam no lar, $24(70,6 \%)$ recebiam até 1 salário mínimo e 32 (94,1\%) moravam em Fortaleza, com 15 famílias morando na regional VI $(44,1 \%)$.

Foram verificados com a análise dos prontuários os valores de peso e estatura ao nascer e atual de cada criança, para que fosse possível acompanhar a sua evolução e ter uma visão global do estado nutricional em que atualmente se encontravam. Para facilitar o entendimento dos dados foram utilizados valores médios das variáveis citadas, obtendo: 2.359 gramas e 40 centímetros ao nascer, 15.998 gramas atual e 104 centímetros atual. Com relação ao estado nutricional das crianças, verificou-se que das 34 participantes da pesquisa, $28(82,4 \%)$ obtiveram o restabelecimento do quadro nutricional e evoluíram para o peso adequado para a idade, porém 5 (14,7\%), mesmo com os acompanhamentos disponíveis no programa, ainda apresentaram um baixo peso para a idade e 1 (2,9\%) apresentou muito baixo peso para a idade.

Destaca-se, então, que todas as crianças convidadas a participar deste estudo foram bebês nascidos com baixo peso $(100 \%, 34)$ e em sua totalidade foram encaminhados para acompanhamento especializado. Nem todas as crianças participantes, no entanto, atingiram até o final do nosso estudo um resultado com $100 \%$ de eficácia permanente. Algumas das participantes, 6 crianças da amostra $(17,6 \%)$, apresentaram alterações de peso com oscilações de perdas e ganhos na pesagem após sair da condição de risco do baixo peso, não atingindo o perfil ideal cronológico, mesmo com o rigor do programa no Iprede. Ao ser realizada a avaliação do perfil psicomotor, observou-se que 20 crianças $(58,9 \%)$ apresentaram o perfil dispráxico, 12 crianças $(35,2 \%)$ o perfil normal e 2 crianças $(5,9 \%)$ o perfil bom, havendo variações das pontuações em cada fator psicomotor analisado.

Para facilitar o entendimento dos resultados, os sete fatores psicomotores foram categorizados de acordo com a prevalência das pontuações (que variam de 1 a 4) e expostos em uma tabela:

Tabela 1 - Frequência das pontuações dos fatores psicomotores

\begin{tabular}{|c|c|c|}
\hline Fatores & $\mathbf{N}$ & $\%$ \\
\hline \multicolumn{3}{|l|}{ Tonicidade } \\
\hline Pontuação 1 e 2 & 4 & 11,8 \\
\hline Pontuação 3 e 4 & $30 *$ & 88,2 \\
\hline \multicolumn{3}{|l|}{ Equilibração } \\
\hline Pontuação 1 e 2 & $25^{*}$ & 73,5 \\
\hline Pontuação 3 e 4 & 9 & 26,5 \\
\hline \multicolumn{3}{|l|}{ Lateralização } \\
\hline Pontuação 1 e 2 & $17^{*}$ & 50,0 \\
\hline Pontuação 3 e 4 & 17 & 50,0 \\
\hline \multicolumn{3}{|l|}{ Noção corpo } \\
\hline Pontuação 1 e 2 & $24^{*}$ & 70,6 \\
\hline Pontuação 3 e 4 & 10 & 29,4 \\
\hline \multicolumn{3}{|c|}{ Estruturação espaçotemporal } \\
\hline Pontuação 1 e 2 & $28^{*}$ & 82,4 \\
\hline Pontuação 3 e 4 & 6 & 17,6 \\
\hline \multicolumn{3}{|l|}{ Praxia global } \\
\hline Pontuação 1 e 2 & $27^{*}$ & 79,4 \\
\hline Pontuação 3 e 4 & 7 & 20,6 \\
\hline \multicolumn{3}{|l|}{ Praxia fina } \\
\hline Pontuação 1 e 2 & $25^{*}$ & 73,5 \\
\hline Pontuação 3 e 4 & 9 & 26,5 \\
\hline
\end{tabular}


Na comparação da pontuação dos fatores psicomotores com o perfil apresentado pode-se observar que, com exceção da tonicidade, todos os outros seis fatores obtiveram valores significativos da prevalência de pontuação 1 e 2 com o perfil dispráxico, como pode ser observado na Tabela 2:

Tabela 2 - Fatores psicomotores e perfil psicomotor

\begin{tabular}{|c|c|c|c|c|}
\hline & \multirow[b]{2}{*}{$\begin{array}{l}\text { Total } \\
\text { n(\%) }\end{array}$} & \multicolumn{2}{|c|}{ Perfil } & \multirow[b]{2}{*}{ P-valo } \\
\hline & & $\begin{array}{c}\text { Bom/ } \\
\text { normal } \\
\text { n(\%) }\end{array}$ & $\begin{array}{l}\text { Dispráxico } \\
\text { n(\%) }\end{array}$ & \\
\hline \multicolumn{5}{|l|}{ Tonicidade } \\
\hline Pontuação 1 e 2 & $(11,8)$ & $0(0)$ & $4(21,1)$ & 0,113 \\
\hline Pontuação 3 e 4 & $30(88,2)$ & $15(100,0)$ & $15(78,9)$ & \\
\hline \multicolumn{5}{|l|}{ Equilibração } \\
\hline Pontuação 1 e 2 & $25(73,5)$ & $6(40,0)$ & $19(100,0)^{*}$ & $<0,001$ \\
\hline Pontuação 3 e 4 & $9(26,5)$ & $9(60,0)^{*}$ & $0(0)$ & \\
\hline \multicolumn{5}{|l|}{ Lateralização } \\
\hline Pontuação 1 e 2 & $17(50,0)$ & $0(0)$ & $17(89,5)^{*}$ & $<0,001$ \\
\hline Pontuação 3 e 4 & $17(50,0)$ & $15100,0)^{*}$ & $2(10,5)$ & \\
\hline \multicolumn{5}{|l|}{ Noção Corpo } \\
\hline Pontuação 1 e 2 & $24(70,6)$ & $5(33,3)$ & $19(100,0)^{*}$ & $<0,001$ \\
\hline Pontuação 3 e 4 & $10(29,4)$ & $10(66,7)^{*}$ & $0(0)$ & \\
\hline \multicolumn{5}{|l|}{$\begin{array}{l}\text { Estruturação } \\
\text { espaçotemporal }\end{array}$} \\
\hline Pontuação 1 e 2 & $28(82,4)$ & $9(60,0)$ & $19(100,0)^{*}$ & 0,002 \\
\hline Pontuação 3 e 4 & $6(17,6)$ & $6(40,0)^{*}$ & $0(0$ & \\
\hline \multicolumn{5}{|l|}{ Praxia global } \\
\hline Pontuação 1 e 2 & $27(79,4)$ & $8(53,3)$ & $19(100,0)^{*}$ & 0,001 \\
\hline Pontuação 3 e 4 & $7(20,6)$ & $7(46,7)^{*}$ & $0(0)$ & \\
\hline \multicolumn{5}{|l|}{ Praxia fina } \\
\hline Pontuação 1 e 2 & $25(73,5)$ & $6(40,0)$ & $19(100,0)^{*}$ & $<0,001$ \\
\hline Pontuação 3 e 4 & $9(26,5)$ & $9(60,0)^{*}$ & $0(0)$ & \\
\hline
\end{tabular}

Fonte: Elaborada pelos autores. Fortaleza - CE, 2018.

Foi realizada uma comparação das variáveis relacionadas aos dados socioeconômicos dos responsáveis pelas crianças com o tipo de perfil apresentado ao final da BPM, e verificado um valor significante na variável da escolaridade e o perfil dispráxico, como pode ser verificado na Tabela 3.

Tabela 3 - Variáveis socioeconômicas e o perfil psicomotor

\begin{tabular}{lccccr}
\hline & & \multicolumn{2}{c}{ Perfil } & \\
\cline { 3 - 4 } & $\begin{array}{c}\text { Total } \\
\mathbf{n}(\%)\end{array}$ & $\begin{array}{c}\text { Bom/ } \\
\text { normal } \\
\mathbf{n}(\%)\end{array}$ & $\begin{array}{c}\text { Disprá- } \\
\text { xico } \\
\mathbf{n}(\%)\end{array}$ & P-valor \\
\hline Escolaridade & & & & \\
$\quad$ Fundamental & $11(32,4)$ & $3(20,0)$ & $8(42,1)^{*}$ & 0,009 \\
Médio incompleto & $12(35,3)$ & $3(20,0)$ & $9(47,4)^{*}$ & \\
Médio completo & $11(32,4)$ & $9(60,0) *$ & $2(10,5)$ &
\end{tabular}

$\begin{array}{lcccc}\begin{array}{l}\text { Profissão } \\ \text { Do lar }\end{array} & 25(73,5) & 11(73,3) & 14(73,7) & 1,000 \\ \quad \text { Outros } & 9(26,5) & 4(26,7) & 5(26,3) & \\ \begin{array}{l}\text { Renda } \\ \text { Até 1 salário mínimo }\end{array} & 24(70,6) & 11(73,3) & 13(68,4) & 1,000 \\ \begin{array}{l}\text { Entre 1 e 3 salários } \\ \text { mínimos }\end{array} & 10(29,4) & 4(26,7) & 6(31,6) & \end{array}$

${ }^{*} p<0,05$

Fonte: Elaborada pelos autores. Fortaleza - CE, 2018.

Como citado anteriormente, 28 (82,4\%) das crianças obtiveram o restabelecimento do quadro nutricional e evoluíram para o peso adequado para a idade, porém $5(14,7 \%)$, mesmo com os acompanhamentos disponíveis no programa, ainda apresentaram um baixo peso para a idade. Na busca de acompanhar essa evolução e verificar o desenvolvimento das crianças, foi feita a comparação do perfil nutricional com o perfil psicomotor e constatado que, mesmo com o peso adequado para a idade, $16(57,1 \%)$ crianças ainda apresentavam o perfil dispráxico, como mostra a Tabela 4:

Tabela 4 - Correlação entre perfil nutricional e perfil psicomotor

\begin{tabular}{lcccc}
\hline & & \multicolumn{2}{c}{ Perfil psicomotor } \\
\cline { 3 - 5 } Perfil nutricional & Total & $\begin{array}{c}\text { Bom/normal } \\
\mathbf{n}(\%)\end{array}$ & $\begin{array}{c}\text { Dispráxico } \\
\mathbf{n}(\%)\end{array}$ \\
\hline Baixo peso para idade & 5 & $2(40,0)$ & $3(60,0)$ \\
Muito baixo peso para idade & 1 & - & $1(100)$ \\
Peso adequado para idade & 28 & $12(42,8)$ & $16(57,1)$ \\
\hline
\end{tabular}

Fonte: Elaborada pelos autores. Fortaleza - CE, 2018.

\section{DISCUSSÃO}

De acordo com os dados obtidos, foi verificada uma pequena diferença com relação ao gênero das crianças, havendo maior prevalência do gênero feminino, corroborando o estudo de Lucena et al. (2010), o qual avalia o desenvolvimento psicomotor e também encontrou uma maior prevalência do gênero feminino. Foi observado durante a pesquisa que a prevalência dos acompanhantes das crianças era de mães, o que é confirmado por Teles (2009) em uma revisão literária sobre a mãe-acompanhante, na qual a autora afirma que a mãe se torna o cuidador principal e modifica a sua rotina para se adequar às necessidades da criança.

Os achados com relação à escolaridade, profissão e renda dos responsáveis foram maiores para os que cursaram o Ensino Médio completo, trabaIhavam no lar e recebiam até 1 salário mínimo/mês, o que corrobora o estudo de Silva et al. (2015) que, 
ao avaliar o perfil nutricional de crianças em unidades de Educação Infantil, obteve que a maioria das mães estudou até o Ensino Médio completo e a profissão de destaque foi a do lar. No que diz respeito à renda familiar, Kucharski et al. (2002), ao avaliarem o perfil de famílias com crianças desnutridas, observaram que $60 \%$ tinham a renda de menos de meio salário mínimo por mês, o que é um dado alarmante e inferior ao que foi visto neste estudo.

Quando verificados os valores médios de peso e estatura ao nascer, foi obtido 2.359 gramas e 40,2 centímetros, respectivamente, estando o peso abaixo da normalidade de acordo com pesquisa de Tourinho e Reis (2013), em que o valor abaixo de 2.500 gramas (baixo peso ao nascer) é considerado um fator importante para a desnutrição e maior probabilidade de riscos nutricionais futuros, o que é destacado ainda por Caçola e Bobbio (2010), segundo os quais as alterações cruciais ocorrem ao longo do desenvolvimento motor, com atrasos e problemas motores específicos. Seguindo o programa do Ministério da Saúde na série Saúde da Criança: acompanhamento do crescimento e desenvolvimento infantil, a estatura média ao nascer deve ser acima de 45 centímetros, o que não foi verificado de forma positiva nos resultados da pesquisa, reafirmando que estavam abaixo da estatura predita como adequada (BRASIL, 2002; GABBARD, 2016).

Com relação ao estado nutricional das crianças, foi constatado que a maioria delas obteve o restabelecimento do quadro nutricional no qual chegaram à instituição, após os acompanhamentos disponíveis no programa de desenvolvimento na primeira infância, o que vai ao encontro da pesquisa de Chuproski et al. (2012) e Goulart, França Júnior e Souza (2009), que afirmam que crianças com déficit nutricional e que são submetidas a programas que visam ao suporte e combate à carência nutricional apresentam resultados significativos de melhora do quadro inicial e uma ressignificação na qualidade de vida.

Apesar de a maioria das crianças terem evoluído no perfil nutricional, algumas ainda apresentam um baixo peso para a idade e necessitam de uma abordagem mais ampla e direcionada para as suas principais necessidades. Conforme afirmado pelo Ministério da Saúde por meio do Relatório do I Seminário sobre Política Nacional de Promoção da Saúde, faz-se necessária uma ação sustentável e articulada para promover ações por parte do Estado que tornem o ambiente mais saudável, possibilitando escolhas alimentares mais factíveis à população, avançando em políticas de saúde e qualidade de vida (BRASIL, 2007, 2013).
Neste estudo foi constatado também que o desenvolvimento da maioria das crianças apresentava-se com déficits (perfil dispráxico) decorrentes da condição nutricional inicial. No que diz respeito ao perfil psicomotor das crianças, a maioria delas apresentaram o perfil dispráxico que "identifica a criança com dificuldades de aprendizagem ligeiras, traduzindo já a presença de um ou mais sinais desviantes, que assume significação neuroevolutiva". De acordo com Fonseca (2012a, p. 105), o aparecimento do padrão dispráxico pode emergir devido a alterações psiconeurológicas relacionadas à maturidade do sistema nervoso central. Nesse sentido, os estímulos e as respostas devem ser integrados e organizados funcionalmente para execução de ações organizadas a partir de movimentos táteis, vestibulares e proprioceptivos.

Durante a comparação dos sete fatores avaliados com o perfil psicomotor, foi observado que, com exceção da tonicidade, todos os outros seis fatores obtiveram a prevalência da pontuação 1 e 2 (fraco e satisfatório, respectivamente), corroborando o encontrado por Fonseca (2012b, p. 266), que afirma ser fácil identificar alterações de equilíbrio, às quais estão associados problemas vestibulares e consequentes sinais de desatenção e impulsividade; alterações de lateralidade, que envolvem a construção simbólica e necessária para que a criança projete o volume do seu corpo no espaço; alterações de noção de corpo, sendo essencial estar preservada para a elaboração de todas as relações com o meio externo; alteração de integração sensorial, não captando, elaborando ou expressando informações, e alterações práxicas em movimentos globais ou finos, que integram todos os outros fatores citados anteriormente e necessários para a regulação e verificação da atividade motora, uma vez que não havendo integração dos fatores, o movimento programado é afetado.

Ressalta-se, portanto, que mediante os resultados obtidos neste estudo, o desenvolvimento psicomotor cronológico durante a primeira infância pode ser prejudicado de forma direta pela baixa condição nutricional, comprometendo as habilidades motoras da criança, além da sua capacidade de se desenvolver física, emocional e cognitivamente pela interferência na maturação do sistema nervoso central (BARROS; MIRANDA; COSTA; 2019).

O resultado obtido na comparação das variáveis socioeconômicas com o tipo de perfil psicomotor vai ao encontro do estudo de Chriarentin et al. (2012), que afirmam que a escolaridade dos pais está intimamente relacionada à sua condição socioeconômica e influencia na moradia, saúde e profissão. O estudo 
também conclui que houve um maior risco de retardo do crescimento e desenvolvimento das crianças cujas famílias tinham uma baixa condição socioeconômica.

Foi observado ainda que houve a melhora do quadro nutricional da maioria das crianças devido à adesão da família ao programa ofertado pelo Iprede. A eficácia conjunta das atividades prestadas pela instituição, no que diz respeito ao acompanhamento, educação em saúde e aconselhamento nutricional somados à intervenção da psicomotricidade, são ferramentas que agem em prol da melhora da qualidade de vida da criança e da família (SILVA et al., 2015).

Com esses dados analisados, foi possível observar que a instituição dedicada a promover acompanhamento no desenvolvimento da primeira infância vem atingindo o seu objetivo com o grupo estudado, comprovado pela evolução da maioria das crianças $(82,4 \%)$ e otimizou a ressignificação do desenvolvimento infantil com redução de sequelas permanentes.

\section{CONCLUSÃO}

A realização deste estudo possibilitou compreender a importância de um acompanhamento multidisciplinar e focado na atenção à saúde da criança, devido aos riscos de prejuízos globais na saúde durante a fase da primeira infância.

Foi possível evidenciar também que, por meio de ações conjuntas envolvendo suporte e acompanhamento nutricional paralelamente à intervenção da psicomotricidade, pôde-se modificar o quadro de baixo peso e favorecer uma intervenção direcionada para o desenvolvimento psicomotor, auxiliando no processo de evolução neuromotora da criança.

Neste estudo foi constatado também que durante o acompanhamento do desenvolvimento psicomotor das crianças com déficits decorrentes da condição nutricional, a participação direta da família no programa, por meio de orientações e educação em saúde, é considerada essencial para uma melhor evolução global do quadro.

Fazem-se necessários mais estudos que abordem a temática do desenvolvimento psicomotor e a eficácia da promoção de saúde no desenvolvimento da primeira infância, incluindo outros fatores, como o tipo de parto, tempo de amamentação exclusiva e de amamentação associada ao leite adaptado. Pensamos que estes fatores também podem influenciar tanto o perfil nutricional como o perfil psicomotor da criança e que vale a pena estudá-los conjuntamente em futuras pesquisas.

\section{REFERÊNCIAS}

ARAÚJO, B. L. Análise do desenvolvimento neuropsicomotor de crianças de zero a três anos em centros de educação infantil. 2013. Monografia (Especialização em Ciências Biológicas) - Universidade Federal do Paraná, Curitiba, 2013.

BARROS, M. G. F. B.; MIRANDA, J. C.; COSTA, R. C. Uso de jogos didáticos no processo ensino-aprendizagem. Rev. Educação Pública, v. 19, n. 23, out. 2019.

BRASIL. Ministério da Saúde. Secretaria de Atenção à Saúde. Estratégias de promoção da alimentação saudável para o nível local. Brasília: Ministério da Saúde, 2007.

BRASIL. Ministério da Saúde. Secretaria de Políticas de Saúde. Saúde da criança: acompanhamento do crescimento e desenvolvimento infantil. Brasília: Ministério da Saúde, 2002.

BRASIL. Ministério da Saúde. Secretaria de Atenção à Saúde. Política Nacional de Alimentação e Nutrição. Brasília: Ministério da Saúde, 2013.

CAÇOLA, P.; BOBBIO, T. G. Baixo peso ao nascer e alterações no desenvolvimento motor: a realidade atual. Rev. Paul. Pediatr, São Paulo, v. 28, n. 1, p. 70-76, 2010.

CHIARENTIN, K. C. et al. Nível socioeconômico e grau de escolaridade: relação com as oportunidades para o desenvolvimento infantil. Revista Digital, Buenos Aires, v. 17, n. 167, abr. 2012.

CHUPROSKI, P. et al. Práticas alimentares e situação social de famílias de crianças desnutridas. Rev. Esc. Enferm. USP, São Paulo, v. 46, n. 1, p. 52-59, 2012.

DIAS, I. S.; CORREIA, S.; MARCELINO, P. Desenvolvimento na primeira infância: características valorizadas pelos futuros educadores de infância. Revista Eletrônica de Educação, São Paulo, v. 7, n. 3, p. 9-24, 2013.

FERREIRA, T. L.; MARTINEZ, A. B.; CIASCA, S. M. Avaliação psicomotora de escolares do $1^{\circ}$ ano do Ensino Fundamental. Rev. Psicopedagogia, v. 27, n. 83, p. 223-235, 2010.

FONSECA, V. da. Desenvolvimento psicomotor e aprendizagem. Porto Alegre: Artmed, 2008.

FONSECA, V. da. Fatores psicomotores e sua relação com as três unidades funcionais. In: FONSECA, V. da. Manual de observação psicomotora: significação psiconeurológica dos fatores psicomotores. Rio de Janeiro: Wak Editora, 2012a. Cap. 3.

FONSECA, V. da. A criança dispráxica com dificuldades de aprendizagem. In: FONSECA, V. da. Manual de observação psicomotora: significação psiconeurológica dos fatores psicomotores. Rio de Janeiro: Wak Editora, 2012b. Cap. 5.

GABBARD, C. Lifelong. Motor Development. 7. ed. Austin: Wolters Kluwer Health, 2016.

GOULART, R. M. M.; FRANÇA JÚNIOR, I.; SOUZA, M. F. M. Fatores associados à recuperação nutricional de crianças em programa de suplementação alimentar. Rev. Bras. Epidemiol, v. 12, n. 2, p. 180-94, 2009.

INSTITUTO DA PRIMEIRA INFÂNCIA - Iprede. 2017. Disponível em: http://www.iprede.org.br/. Acesso em: 11 nov. 2017. 
JARDIM, M. B. Atividades psicomotoras e de integração social em locais de risco e vulnerabilidade social. Rev. Educação Pública, Buenos Aires, v. 20, n. 209, out. 2015.

KUCHARSKI, F. A. et al. Perfil de famílias com crianças desnutridas internadas em uma instituição na cidade de Fortaleza-Ceará. Rev. Rene, Fortaleza, v. 3, n. 2, p. 9-13, jul./dez. 2002.

LUCENA, N. M. G. et al. Relação entre perfil psicomotor e estilo de vida de crianças de escolas do município de João Pessoa, PB. Fisioter. Pesq., São Paulo, v. 17, n. 2, p. 124-129, abr./jun. 2010.

MAIA, R. R. P.; SOUZA, J. M. P. Fatores associados ao baixo peso ao nascer em município do Norte do Brasil. Rev. Bras. Crescimento Desenvolvimento Hum., v. 20, n. 3, p. 735-744, 2010.

MAYER, G. N.; CANCELIER, A. C. L.; FRANCIOTTI, D. L. Comparação do crescimento de bebês com baixo peso ao nascer com bebês nascidos com peso adequado: estudo de coorte. Rev. ACM, Florianópolis, v. 40, n. 4, 2011.

OLIVEIRA, G. E.; MAGALHÃES, L. C.; SALMELA, L. F. T. Relação entre muito baixo peso ao nascimento, fatores ambientais e o desenvolvimento motor e o cognitivo de crianças aos 5 e 6 anos. Rev. Bras. Fisioter, São Carlos, v. 15, n. 2, p. 138-145, mar./abr. 2011.

PNUD. Desenvolvimento Humano e IDH. 2017. Disponível em: http://www.br.undp.org/content/brazil/pt/home/idh0. html. Acesso em: 2 nov. 2019.

PORTUGAL, G. Desenvolvimento e aprendizagem na infância. In: CONSELHO NACIONAL DE EDUCAÇÃO (org.). Relatório do estudo - a educação das crianças dos 0 aos 12 anos. Lisboa: Ministério da Educação, 2009.

SILVA, M. S. et al. Estudo nutricional e socioeconômico de crianças em unidades de educação infantil. Saúde, Santa Maria, v. 41, n. 2, p. 183-192, jul./dez. 2015.

TELES, E. N. O cotidiano da mãe-acompanhante de criança com doença crônica. 2009. Dissertação (Mestrado em Psicologia Clínica) - Pontifícia Universidade Católica de São Paulo, São Paulo, 2009.

TOURINHO, A. B.; REIS, L. B. S. M. Peso ao nascer: uma abordagem nutricional. Com. Ciências Saúde, Brasília, v. 22, n. 4, p. 19-30, 2013.

WILLRICH, A. et al. Desenvolvimento motor na infância: influências dos fatores de risco e programas de intervenção. Rev. Nerocienc., São Paulo, v. 1, n. 17, p. 51-56, 2009. 